\title{
Yeni Piridin Halkası Taşıyan SNS Pincer Tipi Ligand ile Cu, Ni ve Pd Komplekslerinin Sentezi, Karakterizasyonu ve Termal Çalışmaları
}

\author{
Güler DEGER ${ }^{1}$ (D) Hatice Gamze SOGUKOMEROGULLARI*2®, Mehmet SÖNMEZ ${ }^{3}$ (i) \\ 1,3Gaziantep Üniversitesi, Fen-Edebiyat Fakültesi, Kimya Bölümü, 27310, Gaziantep, Türkiye \\ ${ }^{2}$ Gaziantep Üniversitesi, Sağlık Hizmetleri MYO, Tıbbi Hizmetler ve Teknikleri Bölümü, 27310, Gaziantep, \\ Türkiye
}

(Alınış / Received: 29.01.2021, Kabul / Accepted: 01.06.2021, Online Yayınlanma / Published Online: 15.08.2021)

Anahtar Kelimeler

SNS Pincer tip ligand, Metal kompleks,

Karakterizasyon,

Tiyoeter
Özet: 2,6-biskloropiridin ve 2-triflorometilbenzentiyol'ün 1:2 oranında reaksiyonundan yeni SNS pincer tip ligand olan 2,6bis(((triflorometil)fenil)tiyo)metil)piridin (SNS) sentezlenmiştir. Ligand ve sırasıyla $\mathrm{Cu}(\mathrm{II}), \mathrm{Ni}(\mathrm{II})$ ve $\mathrm{Pd}(\mathrm{II})$ klorür tuzlarının 1:1 oranında reaksiyonundan bakır (SNS-Cu(II)), nikel (SNS-Ni(II)) ve palladyum (SNS-Pd(II)) kompleksleri sentezlenmiştir. Komplekslerin merkezinde bulunan metalin (M), hem azot (N) atomuna hem de iki taraftan kükürt (S) atomlarına meridyonel olarak bağlanması ile SNS kompleksleri elde edilmiştir. Sentezlenen bileşikler NMR (sadece ligand), UV-Vis, FT-IR spektroskopik teknikleri, elementel analiz, TGA-DTA, erime noktasi tayini yöntemi ile karakterize edilmiştir. Kompleksler beş koordinasyonlu üçgen bipiramidal geometrik yapıya sahiptir. Ayrıca komplekslerin molar iletkenlik değerlerinin 1.07-1.62 $\mu \mathrm{S} / \mathrm{cm}$ arasında olduğu görülmüștür. Kompleksler iletkenlik özelliğine sahip değildir. Komplekslerin TGA eğrileri incelendiğinde, termal kararlılıklarının $\mathrm{Pd}>\mathrm{Cu}>\mathrm{Ni}$ olduğu gözlemlenmiştir.

\section{Synthesis, Characterization and Termal Studies of $\mathrm{Cu}$, $\mathrm{Ni}$ and Pd Complexes with New SNS Pincer Type Ligand Bearing Pyridine Ring}

Keywords

SNS Pincer type ligand,

Metal complex,

Characterization,

Thioether

\begin{abstract}
The new SNS pincer type ligand 2,6bis(((trifluoromethyl)phenyl)thio)methyl)pyridine (SNS) was synthesized from the reaction of 2,6-bischloropyridine and 2-trifluoromethylbenzenethiol at a ratio of 1:2. Copper (SNS-Cu(II)), nickel (SNS-Ni(II)) and palladium (SNS-Pd(II)) complexes were synthesized from the 1:1 reaction of ligand and $\mathrm{Cu}(\mathrm{II}), \mathrm{Ni}(\mathrm{II})$, Pd(II) chloride salts, respectively. SNS complexes were obtained by meridional bonding of the metal (M) in the center of the complexes to both the nitrogen (N) atom and two sulfur (S) atoms. The synthesized compounds were characterized by NMR (ligand only), UV-Vis, FT-IR spectroscopic techniques, elemental analysis, TGA-DTA, melting point determination method. The complexes have a five coordinated triangular bipyramidal geometric structure. In addition, the molar conductivity values of the complexes were observed to be between 1.07-1.62 $\mu \mathrm{S} / \mathrm{cm}$. The complexes do not have conductivity properties. When the TGA curves of the complexes were examined, it was observed that their thermal stability was $\mathrm{Pd}>\mathrm{Cu}>\mathrm{Ni}$.
\end{abstract}

\section{Giriș}

1970'lerden itibaren pincer ligand ve kompleksleri üzerine çalışmalar yapılmaya başlanmış ve bu bileşiklerin doğası açıklanmaya çalışılmıștır. Pincer bileşiklerinin katalitik, farmasötik, biyokimya gibi bir çok disiplinde ilgi çekici ve önemli çalışmalarda da etkili olduğu vurgulanmıştır [1,2]. Geçiş metallerinin çeşitliliği sayesinde pincer(kıskaç) tipinde birçok liganddan farklı kompleksler sentezlenmiştir. Bu sentez çalışmaları liganddaki çeşitliliği desteklemekte ve halen araștırmalar devam etmektedir [3-6].

Kıskaç komplekslerin yapısı incelendiğinde, iyi termal stabiliteleri ve ayarlanabilir kimyasal özellikleri avantaj sağlamaktadır. Pincer komplekslerin azot (N), oksijen (O), fosfor (P), kükürt (S) ve karbon (C) gibi elektron verici atomlar ve alkil, aril grupları gibi yer değiştiren gruplarla çevrelenmesiyle kazandığı elektronik ve sterik 
özellikleri sayesinde, metal boyutunun tekrar değiştirilmesi sağlanmaktadır [7-10].

Aromatik bileșiklerden türeyen pincer ligand ve kompleksleri üzerine yapılan çalışmalar gün geçtikçe daha da ilgi çekici olmaktadır. Aromatik pincer ligandlar merkezdeki metal için katı bir koordinasyon ortamı sağlamakta ve böylelikle yüksek termal kararlılığa sahip yeni pincer kompleksler oluşmaktadır [11].

Kıskaç ligandların özellikleri son yıllarda göz alıcı olmaya başlamıştır. Bu önemli özelliklerden yararlanılan katalizörlerin; özellikle geri kazanılabilirlik ve tekrar kullanılabilirlik, katı destekler üzerindeki immobilizasyonu son zamanlarda daha geniş değerlendirme imkanı sağlamıştır [12]. Bu açıdan bakıldığında; silika, manyetik, nanopartiküller, karbon nanotüpler, reçineler, polimerler gibi çeşitli katı destekler ve MOF(molekül organik kafesler), çok yönlü metal kıskaç katalizörlerinin hareketsizleştirilmesi için kullanılmıştır [13-19].

SNS ligand kompleksleri diğer tipteki pincer(kıskaç) komplekslere göre daha az ilgi görmüştür. Etilenin trimerizasyonunda SNS-Cr katalizörünün iyi aktivite göstermesiyle SNS tipindeki komplekslere dayalı araştırmalar artmıştır $[20,21]$. Tridentat SNS ligandlarının başka bir avantajı ligandları değișime, çeşitliliğe açık hale getiren omurgaların karışık donör mukavemetidir. SNS kompleksleri katalitik uygulamalar açısından oldukça ilgi çekmektedir. Dahası SNS-Cu(II) kompleksleri üzerine de birçok çalışma rapor edilmiștir [22-24].

$\mathrm{Bu}$ çalışmada, yeni SNS tipindeki 2,6bis(((triflorometil)fenil)tiyo)metil)piridin ligand ve bu ligandin $\mathrm{Cu}^{+2}, \mathrm{Pd}^{+2}$ ve $\mathrm{Ni}^{+2}$ kompleksleri sentezlenmiştir. Sentezlenen ligand ve komplekslerin yapıları; elementel analiz, FT-IR, UV-Vis, NMR, TGA gibi yöntemlerle aydınlatılmıştır.

\section{Materyal ve Metot}

\subsection{Materyal}

Deneysel çalışmalarda laboratuar ortamında kullanılan tüm kimyasallar, saflaştırma ve sentez için kullanılan çözücüler, Sigma-Aldrich ve Merck'in Türkiye'deki distribütör firmalarından sağlanmıştır. DMF içerisinde ligandın ve kompleks bileşiklerin ultraviyole-görünür (UV-Vis) spektrumları PG Instruments (UK) T80 + UV-Vis spektrofotometre ile kaydedilmiştir. Ligandın ${ }^{1} \mathrm{H}$ ve ${ }^{13} \mathrm{C}$ nükleer manyetik rezonans (NMR) spektrumları, Bruker Yüksek Performanslı Dijital FTNMR (400 MHz $)$ spektrometresi kullanılarak DMSO-d6'da ölçülmüştür. Bileşiklerin FT-IR spektrumları, Perkin Elmer Spectrum 100 FT-IR Spektrometresi (ATR örnekleme aksesuarı) kullanılarak 4000-400 $\mathrm{cm}^{-1}$ aralığında ölçülmüştür. C, H, N ve S için element analizi Thermo Scientific Flash EA 2000 CHNS elementel analiz cihazı ile yapılmıștır. TGA eğrileri STA7300 HITACHI cihazı ile azot gazı $\left(\mathrm{N}_{2}\right)$ ortamında $20-1000{ }^{\circ} \mathrm{C}$ aralığında ve dakikada $10{ }^{\circ} \mathrm{C}$ artırılarak yakılması kaydedilmiştir. Molar iletkenlik ölçümleri, oda koşullarında ve DMF de ORION 4 STAR pH Conductivity Benchtop model iletkenlik cihazıyla ölçülmüștür.

\subsection{Metot}

\subsubsection{2,6-bis(((triflorometil)fenil)tiyo)metil) piridin ligandın sentezi (SNS)}

0,177 g 2,6-biskloropiridin üzerine $20 \mathrm{ml}$ DMF ilave edilip çözülmesi sağlandı. Üzerine $0,166 \mathrm{~g}$ KI ve 0,542 $\mathrm{g} \mathrm{K}_{2} \mathrm{CO}_{3}$ üzerine eklendi. Aynı balonun içerisine 264 $\mu \mathrm{l} 2$-triflorometilbenzentiyol eklendi. Balon, azot gazı ortamında $80{ }^{\circ} \mathrm{C}$ sicaklıkta 48 saat boyunca yağ banyosunda isıtıldı. Sentezlenen madde buz içerisine koyularak tepkime sonlandırıldı. Üzerine $15 \mathrm{ml}$ kloroform ilave edildi. Sentez ürünü organik faza çekildi. Reaksiyon ortamında çözücü evaporatör yardımıyla uzaklaştırıldı ve ürün kurutuldu. Ligand THF de çözüldü ve diş ortama dietileter konularak yavaş difüzyon yöntemiyle saflaştırıldı. Viskoz, yağımsı bir ligand elde edildi (Şekil 1). (90.4 \%); IR, (ATR) cm¹: 3066 (C-H) aromatik, 2933 (C-H)alifatik, 1571 $(\mathrm{C}=\mathrm{N})_{\text {piridin, }} 1258 \quad(\mathrm{C}=\mathrm{N}-\mathrm{C}), 757$ (C-S-C); ${ }^{1} \mathrm{H}-\mathrm{NMR}$ DMSO- $\left.\mathrm{d}_{6}, 400 \mathrm{MHz}\right): \delta 7.83(\mathrm{t}, 1 \mathrm{H}, \mathrm{J}=8 \mathrm{~Hz}, \mathrm{H}-4), 7.71$ $\left(\mathrm{d}_{\mathrm{d}}, 4 \mathrm{H}, \mathrm{H}-11, \mathrm{H}-10\right), 7.54(\mathrm{t}, 2 \mathrm{H}, \mathrm{J}=8 \mathrm{~Hz}, \mathrm{H}-9), 7.34\left(\mathrm{~d}_{\mathrm{d}}\right.$, $4 \mathrm{H}, \mathrm{H}-8, \mathrm{H}-3$ ) , 4.42 (s, 4H, H-5), 3.36 çözücü piki, 2.50 su piki. ${ }^{13} \mathrm{C}-\mathrm{NMR}$ (DMSO-d6,400 MHz): $\delta 156.68(\mathrm{C}=\mathrm{N}$ ) C-2 karbonu, 138.27 C-4 karbonu, 136.20 C-10 karbonu, 133.14 C-7 karbonu, 130.42 C-6 karbonu, 127.18 C-11 karbonu, 127.12 C-9 karbonu, 127.05 C8 karbonu, 126.35 C-3 karbonu, 122.30 C-12 karbonu, 39.01 C-5 karbonu; UV-Vis (DMF) $\lambda \max$ (Abs): 270 (1,901), 277.38 ( 1.399 ) nm. İletkenlik $2 \times 10^{-5} \mathrm{M}$ DMF $\left(\Lambda_{\mathrm{M}}\right): 0.85 \mu \mathrm{S} / \mathrm{cm}$. Teorik: $\mathrm{C}_{21} \mathrm{H}_{15} \mathrm{~F}_{6} \mathrm{NS}_{2}$ (459.47): C, 54.89; H, 3.29; N, 3.05; S, 13.96\%. Bulunan: C, 54.63; H, \%3.60; N, \%3.74; S, 13.39\%.

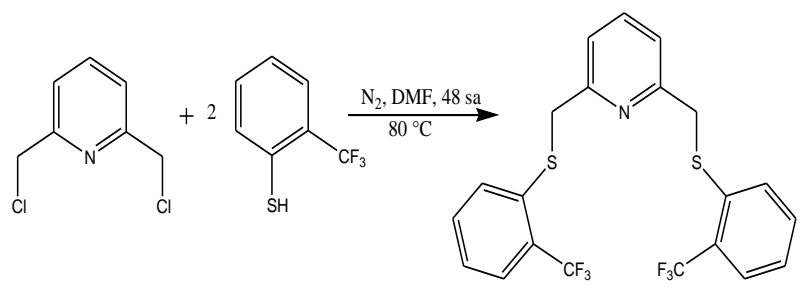

Şekil 1. Ligandın Genel Sentez Şeması

\subsubsection{Komplekslerin genel sentez prosedürü}

1 mmol ligandın üzerine $15 \mathrm{ml}$ THF ilave edilerek 60 ${ }^{\circ} \mathrm{C}$ sıcaklıkta çözüldü. Üzerine $10 \mathrm{ml}$ metanol içerisinde çözünen $1 \mathrm{mmol}$ metal klorür tuzu ilave edildi. Aniden renk dönümü gerçekleşti. 2 saat boyunca ISı ortamında karıştırıldı Çökme gerçekleşince tepkime tamamlandı. Daha sonra elde edilen madde süzüldü, soğuk su, metanol ve 
dietileterde yıkandı. Etanolde saflaştırıldı ve desikatörde kurutuldu (Şekil 2).

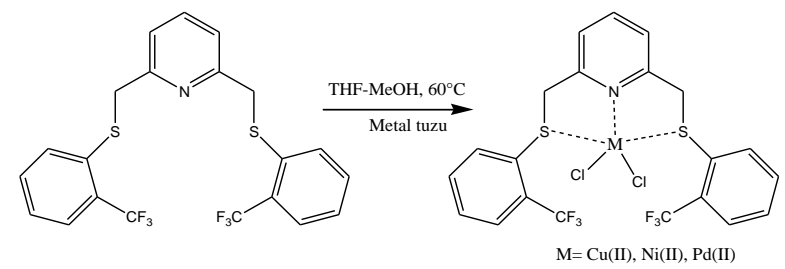

Şekil 2. Kompleklerinin Genel Sentez Şeması

\subsubsection{1. [ $\left.\mathrm{Cu}\left(\mathrm{\kappa}^{3}-\mathrm{SNS}\right) \mathrm{Cl}_{2}\right] \cdot \mathrm{H}_{2} \mathrm{O}$ kompleksinin sentezi (SNS-Cu)}

Oluşan $\mathrm{Cu}(\mathrm{II})$ kompleksinin rengi koyu yeşildir. Verim: 0.452 g (76.2 \%). E.N: $95-96{ }^{\circ} \mathrm{C}$. IR, (ATR) $v$, cm-1: $3263(\mathrm{O}-\mathrm{H}), 3068$ (C-H) aromatik, 2960 (C-H)alifatik, $1572(\mathrm{C}=\mathrm{N})_{\text {piridin, }} 1259(\mathrm{C}=\mathrm{N}-\mathrm{C}), 761$ (C-S-C); UV-Vis (DMF) $\lambda_{\max }$ (Abs): 270 (1,650), 290.44 (0.874), 357.74 (0.052), 301.49 (0.531) nm; İletkenlik 2x10-5 $\operatorname{M~DMF~}\left(\Lambda_{\mathrm{M}}\right): 1.07 \mu \mathrm{S} / \mathrm{cm}$. Teorik: $\mathrm{C}_{21} \mathrm{H}_{17} \mathrm{Cl}_{2} \mathrm{CuF}_{6} \mathrm{NOS}_{2}$ (611.93); C, 41.22; H, 2.80; N, 2.29; S, $10.48 \%$. Bulunan: C, 42.09; H, 3.17; N, 2.99; S, $10.59 \%$.

\subsubsection{2. $\quad\left[\mathrm{Ni}\left(\kappa^{3}-\mathrm{SNS}\right) \mathrm{Cl}_{2}\right] \cdot 2 \mathrm{H}_{2} \mathrm{O} \quad$ kompleksinin sentezi (SNS-Ni)}

Oluşan nikel kompleksinin rengi yeşil-sarıdır. Kompleks yağımsı viskoz bir yapıdadır. Verim: 0.556 g (89.1 \%). IR, (ATR) v, cm-1: $3312(\mathrm{O}-\mathrm{H}), 3067$ (CH) aromatik, 2967 (C-H) alifatik, 1572 (C=N) piridin, 1258 $(\mathrm{C}=\mathrm{N}-\mathrm{C}), 758$ (C-S-C); UV-Vis (DMF) $\lambda_{\max }(\mathrm{Abs}): 270$

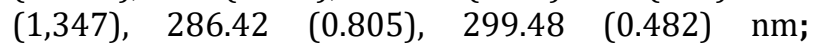
İletkenlik $2 \times 10^{-5} \mathrm{M}$ DMF $\left(\Lambda_{\mathrm{M}}\right): 1.27 \mu \mathrm{S} / \mathrm{cm}$. Teorik: $\mathrm{C}_{21} \mathrm{H}_{19} \mathrm{Cl}_{2} \mathrm{~F}_{6} \mathrm{NNiO}_{2} \mathrm{~S}_{2}$ (625.1); C, 40.35; H, 3.06; N, 2.24; S, $10.26 \%$. Bulunan: C, 40.35; H, \%2.91; N, \%2.40; S, $\% 9.82 \%$.

\subsubsection{3. [ $\left.\mathrm{Pd}\left(\mathrm{\kappa}^{3}-\mathrm{SNS}\right) \mathrm{Cl}_{2}\right] \cdot 3 \mathrm{C}_{3} \mathrm{H}_{7} \mathrm{NO}$ kompleksinin sentezi (SNS-Pd)}

Oluşan paladyum kompleksinin rengi bordodur. Verim: 0.558 g (65.3 \%). E.N: $78-79{ }^{\circ}$ C. IR, (ATR) $v$, $\mathrm{cm}^{-1}: 3065$ (C-H) aromatik, 2959 (C-H) alifatik, 1571 $(\mathrm{C}=\mathrm{N})$ piridin, 1257 (C=N-C), 764 (C-S-C); UV-Vis (DMF) $\lambda_{\max }$ (Abs): 270 (1,245), 285.42 (1.002), 300.49 (0.805), 351.71 (0.243), $400.93 \quad$ (0.201) nm; İletkenlik $2 \times 10^{-5} \mathrm{M}$ DMF $\left(\Lambda_{\mathrm{M}}\right): 1.62 \mu \mathrm{S} / \mathrm{cm}$. Teorik: $\mathrm{C}_{30} \mathrm{H}_{36} \mathrm{Cl}_{2} \mathrm{~F}_{6} \mathrm{~N}_{4} \mathrm{O}_{3} \mathrm{PdS}_{2}$ (856.08); C, 42.09; $\mathrm{H}, 4.66 ; \mathrm{N}$, 6.65; S, 7.49 \%. Bulunan: C, 42.09, H, 4.24, N, 6.54, S, $6.69 \%$.

\section{Bulgular ve Tartışma}

Ligand, 2,6-biskloropiridin ve 2(triflorometil)benzentiyol'un 1:2 oranında tepkimesinden sentezlenmiştir. Genel sentez şeması Şekil 1'de gösterildiği gibidir. Bu teknikle 2,6-bis(c(2triflorometil)fenil)tiyo)metil)piridin SNS ligandı sentezlenmiştir. Sentezlenen ligand orijinal olup literatür bilgileriyle uyum sağlamaktadır. Bu liganddan elde edilen SNS-Cu, SNS-Ni ve SNS-Pd komplekleri Şekil 2'de gösterilen tepkime şemasına göre sentezlenmiştir. Liganda ve kompleks bileşiklere ait sonuçlar; molekül ağırlıkları, renkleri, molekül formülleri, fiziksel görünümleri ve elementel analiz sonuçları Bölüm 2.2. Yöntemler bölümünde belirtildiği gibi olup literatür bilgileri ile uyum içerisindedir [24]. SNS pincer tip metal kompleksleri renkli ve havaya dayanıklıdır ayrıca THF, DMF ve etanol gibi yaygın organik çözücüler içinde iyi çözünürler. Komplekslerin molar iletkenlik değerlerinin 1.07-1.62 $\mu \mathrm{S} / \mathrm{cm}$ arasında olduğu görülmüştür. $\mathrm{Bu}$ da metal komplekslerinin elektrolitik iletkenliğinin olmadığını göstermektedir [25].

Bu çalışmanın FT-IR sonuçlarına bakıldığında, ligand ve kompleksler için aromatik $\mathrm{C}$-H gerilme bandı 3065-3068 $\mathrm{cm}^{-1}$, alifatik C-H gerilme bandı 2926$2967 \mathrm{~cm}^{-1}$, piridin halkasına ait $\mathrm{C}=\mathrm{N}$ gerilme band 1571-1572 $\mathrm{cm}^{-1}[26,27]$, piridin halkasina ait $\mathrm{C}-\mathrm{N}=\mathrm{C}$ eğilme bandı 1257-1259 $\mathrm{cm}^{-1}$, C-S-C gerilme bandı $757-764 \mathrm{~cm}^{-1}$ de gözlemlenmiştir. Ligandda $757 \mathrm{~cm}^{-1}$ de görülen C-S-C bandının komplekslerde 758-764 $\mathrm{cm}^{-1}$ de görülmüştür. Gerilme bandındaki 1-7 $\mathrm{cm}^{-1}$ arasındaki kayma kompleksleşmenin buradan gerçekleştiğini göstermektedir [24]. Komplekslerde M-N gerilme band $556-594 \mathrm{~cm}^{-1}[28,29], \mathrm{M}-\mathrm{S}$ gerilme bandı $442-463 \mathrm{~cm}^{-1}$ de rastlanmaktadır [24, 30].

SNS bileșiğinin ${ }^{13} \mathrm{C}-\mathrm{NMR}$ alınan spektrum kaydında 156.68 ppm'de, piridin halkasındaki C-2 karbonuna bağlı C=N sinyaline aittir. 138.27-122.30 ppm aralığındaki sinyal şiddetlerine bileşikteki piridin ve fenil halkalarına ait aromatik karbonlara aittir. Yapıdaki kükürt atomuna bağlı $\mathrm{CH}_{2}$ karbonlarına ait sinyal 39.00 ppm'de rastlanmıştır (Şekil 3). SNS ligand bileşiğinin ${ }^{1} \mathrm{H}-\mathrm{NMR}$ spektrum kaydında 7.83 ppm'de $\mathrm{H}-4$ protonu $1 \mathrm{H}$ triplet olarak, 7.71 ppm'de $\mathrm{H}-11$ ve $\mathrm{H}-10$ protonlari $4 \mathrm{H}$ dubletin dubleti olarak, 7.54 ppm'de $\mathrm{H}-9$ protonu $2 \mathrm{H}$ triplet olarak, $7.34 \mathrm{ppm}$ de $\mathrm{H}-8$ ve $\mathrm{H}-3$ protonu $4 \mathrm{H}$ dubletin dubleti olarak, 4.42 ppm'de $\mathrm{H}-5$ protonu singlet olarak görülmektedir. 3.36 ppm'de çözücüye (DMSO-d6), 2.50 ppm'de sudan gelen sinyal şiddetine rastlanmaktadır (Şekil 4). Bu değerler literatürle uyum içerisindedir.

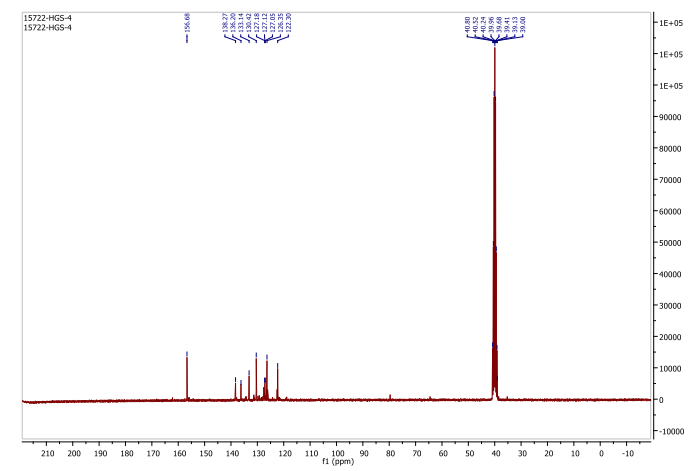

Şekil 3. Ligandın ${ }^{13} \mathrm{C}-\mathrm{NMR}$ spektrumu 


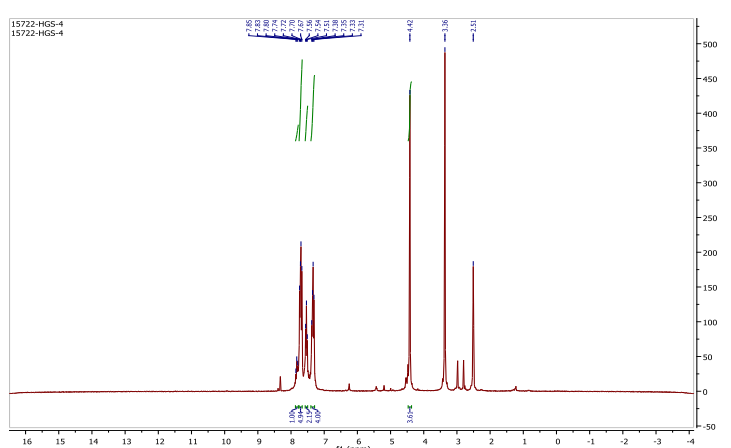

Şekil 4. Ligandın ${ }^{1} \mathrm{H}-\mathrm{NMR}$ spektrumu

Komplekslerin TGA eğrileri incelendiğinde, termal kararlılıklarının $\mathrm{Pd}>\mathrm{Cu}>\mathrm{Ni}$ olduğu gözlemlenmiştir. SNS-Cu eğrisi incelendiğinde, beş basamakta termal bozunum gözlenmiştir. İlk basamakta $1 \mathrm{~mol} \mathrm{H}_{2} \mathrm{O}$ dehidratasyonu [31], ikinci basamakta $2 \mathrm{~mol} \mathrm{HCl}$ çıkışı, üçüncü basamakta $2 \mathrm{~mol}$ triflorometilfenil, dördüncü ve beşinci basamakta ise diğer organik gruplar dekompoze olarak metal okside dönüşmüştür. SNS-Ni eğrisi incelendiğinde, beş basamakta termal bozunum göstermiștir. İlk basamakta 2 mol $\mathrm{H}_{2} \mathrm{O}$ dehidratasyonu [31], ikinci basamakta 2 mol HCl çıkışı, üçüncü basamakta 2 mol triflorometilfenil, dördüncü ve beşinci basamakta ise organik gruplar dekompoze olarak metal okside dönüşmüştür. SNS-Pd eğrisi incelendiğinde, dört basamakta termal bozunum göstermiştir. İlk basamakta $1 \mathrm{~mol} \mathrm{HCl} \mathrm{çlkışı} \mathrm{ve} \mathrm{ikinci} \mathrm{basamakta} 1$ mol $\mathrm{HCl}$ ve 1 mol $\mathrm{C}_{3} \mathrm{H}_{7} \mathrm{NO}$ çıkışı, üçüncü basamakta 2 mol $\mathrm{C}_{3} \mathrm{H}_{7} \mathrm{NO}$ çıkışı ve $2 \mathrm{~mol}$ triflorometilfenil çıkışı, dördüncü basamakta ise diğer organik gruplar dekompoze olarak metal okside dönüșmüștür (Tablo 1). 3 eğri için son sıcaklık aralıklarında olan kütle kaybının, organik parçalamalar ve metal okside ait olduğu düşünülmektedir. Gerçekleşen TGA analiz sonuçları literatürdeki benzer çalışmalarla uyum içerisindedir [32].

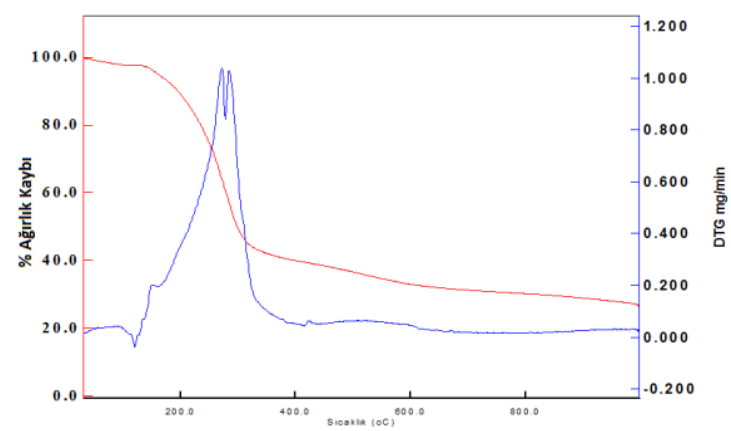

Şekil 5. SNS-Cu kompleksinin TGA eğrisi

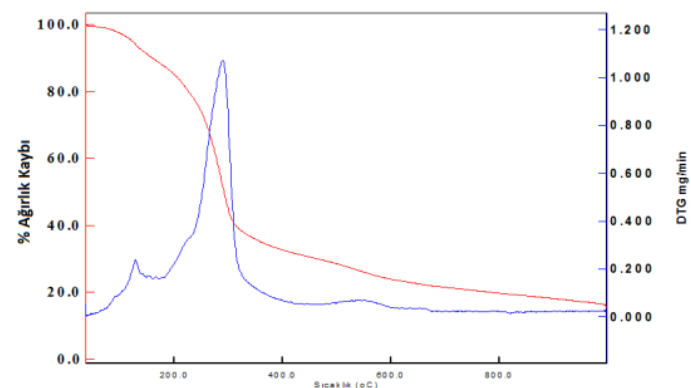

Șekil 6. SNS-Ni kompleksinin TGA eğrisi

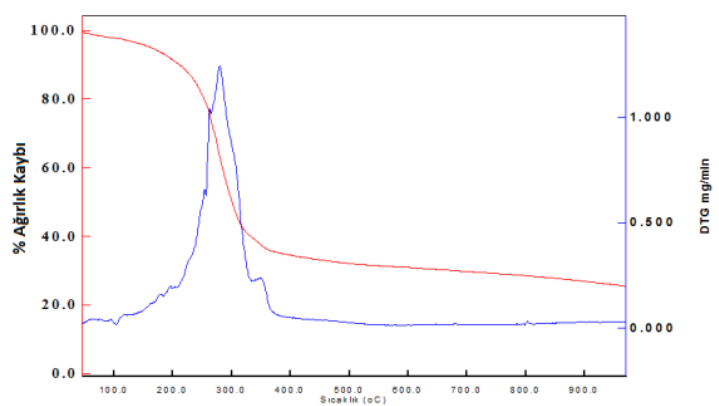

Şekil 7. SNS-Pd kompleksinin TGA eğrisi

Bileşiklerin UV-Vis spektrumları DMF çözücüsünde, 2x10-5 M konsantrasyonda 190-1100 nm aralığında kaydedilmiştir. SNS ligand ve komplekslere ait UV-Vis sonuçlarına bakıldığında hem ligandda hem de SNS(Cu, Pd, Ni) komplekslerinde meydana gelen 270 nm'deki pik; fenil halkasına ait $\pi-\pi^{*}$ geçişlerini göstermektedir. Burada fenil halkasına ait SNS ligandında $277 \mathrm{~nm}$ 'de ki görülen absorbsiyon bandı, SNS-Cu kompleksinde; 290, 301, 357 nm dalga boylarındaki absorbsiyon bandları, SNS-Ni kompleksinde 286, $299 \mathrm{~nm}$ dalga boyundaki absorbsiyon bandları ve SNS-Pd kompleksindeki 285, $300,351 \mathrm{~nm}$ dalga boylarındaki absorbsiyon banlarının, piridin halkasına ait $\pi-\pi^{*}$ ve $n-\pi^{*}$ geçişlerine ait olduğunu göstermektedir. SNS-Cu ve SNS-Ni komplekslerinin UV-Vis sonuçlarında, yük transfer geçişleri net olarak gözlenememiştir.

Bunun maskelenmeden dolayı olduğu düşünülmektedir. SNS-Pd UV-Vis sonuçlarında ise 400 nm'deki absorbsiyon band,, yük transfer geçișlerinin varlığını göstermektedir. Bu da kompleks oluşumuna işaret etmektedir [24, 27].

Sentezlenen SNS komplekslerinin yapisal karakterizasyonu incelendiğinde, komplekslerin üçgen bipiramidal yapıda olduğu düşünülmektedir $[24,30,33,34]$

Tablo 1. Komplekslerin TGA eğrileri (ağırlık kaybı \%; Hesaplanan (Bulunan))

\begin{tabular}{ccccc}
\hline Bileşikler & $\begin{array}{c}\text { Birinci basamak } \\
\text { (\% ağırlık kaybı) }\end{array}$ & $\begin{array}{c}\text { İkinci basamak } \\
\text { (\% ağırlık kaybı) }\end{array}$ & $\begin{array}{c}\text { Üçüncü basamak } \\
\text { (\% ağırlık kaybı) }\end{array}$ & $\begin{array}{c}\text { Dördüncü basamak } \\
\text { (\% ağırlık kaybı) }\end{array}$ \\
\hline \multirow{2}{*}{ SNS-Cu } & $20-147^{\circ} \mathrm{C}$ & $147-217^{\circ} \mathrm{C}$ & $217-311^{\circ} \mathrm{C}$ & $311-606^{\circ} \mathrm{C}$ \\
& $2.94(2.8)$ & $11.92(12)$ & $47.4(45)$ & $10.47(11)$ \\
\multirow{2}{*}{ SNS-Ni } & $20-123^{\circ} \mathrm{C}$ & $123-211^{\circ} \mathrm{C}$ & $211-311^{\circ} \mathrm{C}$ & $311-588^{\circ} \mathrm{C}$ \\
& $5.71(5.5)$ & $11.42(11.5)$ & $46.66(46.5)$ & $14.7(14.8)$ \\
\multirow{2}{*}{ SNS-Pd } & $20-150^{\circ} \mathrm{C}$ & $150-250^{\circ} \mathrm{C}$ & $250-361^{\circ} \mathrm{C}$ & - \\
& $4.26(4)$ & $12.79(13)$ & $51.86(49)$ & - \\
\hline
\end{tabular}




\section{Sonuç}

$\mathrm{Bu}$ çalışmada pincer (kıskaç) bileşiklerinin doğası üzerinde durulmuştur. Gerçekleștirilen çalışmada 2,6-biskloropiridin ve 2-triflorometilbenzentiyol'den türeyen yeni SNS tipi bileşik olarak, 2,6bis(((triflorometil)fenil)tiyo)metil)piridin ligandı ve bu ligandın $\mathrm{Cu}(\mathrm{II}), \mathrm{Pd}(\mathrm{II})$ ve Ni(II) klorür tuzları ile 1:1 sitokiyometrik oranında reaksiyonlarından metal komplekslerinin sentezlenmesi gerçekleştirilmiştir. Elde edilen komplekslerin merkezinde bulunan metalin (M), hem azot $(\mathrm{N})$ atomuna hem de iki taraftan kükürt (S) atomlarına meridyonel olarak bağlanması ile SNS kompleksleri elde edilmiştir. Komplekslerin üçgen bipiramit yapıda olduğu düşünülmektedir.

Elde edilen SNS ligand ve komplekslerin karakterizasyonları, elementel analiz, FT-IR, UV-Vis, NMR (sadece ligand) ve TGA analizleri ile yapılmıștır. Sentezlenen maddeler orijinal olup literatür çalışmalarıyla uyum göstermektedir. Literatürde SNS pincer bileşikleri üzerine yapılan çalışmaların fazla olmamasından dolayı, sentezlenen SNS ligand ve komplekslerinin, pincer bileşiklerinin doğası ile ilgili olan çalışmalara katkı sağlayacağı düşünülmektedir.

\section{Etik Beyanı}

Bu çalıșmada, "Yükseköğretim Kurumları Bilimsel Araștırma ve Yayın Etiği Yönergesi" kapsamında uyulması gerekli tüm kurallara uyulduğunu, bahsi geçen yönergenin "Bilimsel Araştırma ve Yayın Etiğine Aykırı Eylemler" başlı̆̆ı altında belirtilen eylemlerden hiçbirinin gerçekleştirilmediğini taahhüt ederiz.

\section{Kaynakça}

[1] Moulton, C. J., Shaw, B. L. 1976. Transition metal-carbon bonds. Part XLII. Complexes of nickel, palladium, platinum, rhodium and iridium with the tridentate ligand 2,6-bis[(di-tbutylphosphino)methyl] phenyl. Journal of the Chemical Society Dalton Transactions, 11, 10201024.

[2] Van Koten, G., Timmer, K., Noltes, J. G., Spek, A. L. 1978. A novel type of $\mathrm{Pt}-\mathrm{C}$ interaction and a model for the final stage in reductive elimination processes involving $\mathrm{C}-\mathrm{C}$ coupling at $\mathrm{Pt}$; synthesis and molecular geometry of $\left[1, N^{\prime} N^{\prime}-\eta-2,6\right.$ bis $\{$ (dimethylamino) methyl $\}$ toluene]iodoplatinum(II)Tetrafluoroborate. Journal of the Chemical Society, Chemical Communications, 6, 249-250.

[3] Hussein, A. Y., Nazir, A., Wei, S., Francis, V. 2014. Ruthenium pincer complexes: Ligand design and complex synthesis. Coordination Chemistry Reviews, 276, 112-152.

[4] Albrecht, M., Van Koten, G. 2001. Platinum group organometallics based on pincer complexes: sensors, switches, and catalysts in memory of Prof. Dr. Luigi M. Venanzi and his pioneering work in organometallic chemistry, particularly in PCP pincer chemistry. Angewandte Chemie International Edition, 40, 3750-3781.

[5] Van der Boom, M. E., Milstein, D. 2003. Cyclometalated phosphine-based pincer complexes: mechanistic insight in catalysis, coordination, and bond activation. Chemical Reviews, 103, 1759-1792.

[6] Creaser, C. S., Kaska, W. C. 1978. Complexes of 1,3- bis(dimethylphosphinomethyl) benzene with nickel(II), paladium(II) and iron(II) halides. Inorganica Chimica Acta, 30, 325-326.

[7] Empsall, H. D., Hyde, E. M., Markham, R., McDonald, W. S., Norton, M. C., Shaw, B. L., Weeks, B. 1977. Synthesis and X-ray structure of an unusual iridium ylide or carbene complex. Journal of the Chemical Society, Chemical Communications, 589-590.

[8] Kelly, W. S. J., Ford, G. H., Nelson, S. M. 1971. Studies on the magnetic crossover in fivecoordinate complexes of iron(II), cobalt(II), and nickel(II). Part I. Journal of the Chemical Society A: Inorganic, Physical, Theoretical, 388-396.

[9] Moulton, C. J., Shaw, B. L. 1976. Transition metal-carbon bonds. Part XLII. Complexes of nickel, palladium, platinum, rhodium and iridium with the tridentate ligand 2,6-bis[(ditbutylphosphino) methyl]phenyl. Journal of the Chemical Society Dalton Transactions, 10201024.

[10] Khusnutdinova, J. R., Milstein, D. 2015. Metalligand cooperation. Angewandte Chemie International Edition, 54, 12236-12273.

[11] Albrecht, M., Van Koten, G. 2001. Platinum group organometallics based on "pincer" complexes: sensors, switches, and catalysts. Angewandte Chemie International Edition, 40, 3750-3781.

[12] Zohreh, N., Hosseini, S. H., Tavakolizadeh, M., Busuioc, C., Negrea, R. 2018. Palladium pincer complex incorporation onto the $\mathrm{Fe}_{3} \mathrm{O}_{4}$ entrapped cross-linked multilayered polymer as a high loaded nanocatalyst for oxidation. Journal of Molecular Liquid, 266, 393-494.

[13] Rimoldi, M., Fodor, D., Van Bokhoven, J. A., Mezzetti, A. 2015. Catalytic hydrogenation of liquid alkenes with a silica-grafted hydride pincer iridium(III) complex: support for a heterogeneous mechanism. Catalysis Science \& Technology, 5, 4575-4586.

[14] Zeng, T., Yang, L., Hudson, R., Song, G., Moores, A. R., Li, C. J. 2011. $\mathrm{Fe}_{3} \mathrm{O}_{4}$ Nanoparticle-supported Copper(I) pybox catalyst: magnetically recoverable catalyst for enantioselective directaddition of terminal alkynes to imines. Organic Letter, 13, 442-445. 
[15] Zohreh, N., Jahani, M. 2017. NNN-pincer-copper complex immobilized on magnetic nanoparticles as a powerful hybrid catalyst for aerobic oxidative coupling and cycloaddition reactions in water. Journal of Molecular Catalysis A: Chemical, 426, 117-129.

[16] Machado, K., Mishra, J., Suzuki, S., Mishra, G. S. 2014. Synthesis of superparamagnetic carbon nanotubes immobilized $\mathrm{Pt}$ and $\mathrm{Pd}$ pincer complexes: highly active and selective catalysts towards cyclohexane oxidation with dioxygen. Dalton Transactions, 43, 17475-17482.

[17] Tamami, B., Mohaghegh Nezhad, M., Ghasemi, S., Farjadian, F. 2016. Modified merrifield resinsupported PCP pincer palladium nanoparticles as a new polymeric catalyst for cyanation of aryl iodides. Phosphorus, Sulfur, and Silicon and the Related Elements, 191, 123128.

[18] Baran, T., Menteş, A. 2017. Construction of new biopolymer (chitosan)-based pincer-type Pd (II) complex and its catalytic application in Suzuki cross coupling reactions. Journal of Molecular Structure, 1134, 591-598.

[19] Pintado-Sierra, M., Rasero-Almansa, A. M., Corma, A., Iglesias, M., Sánchez, F. 2013. Bifunctional iridium-(2-aminoterephthalate)Zr-MOF chemoselective catalyst for the synthesis of secondary amines by one-pot threestep cascade reaction. Journal of Catalysis, 299, 137-145.

[20] McGuinness, D. S., Wasserscheid, P., Keim, W., Morgan, D., Dixon, J. T., Bollmann, A., Maumela, H., Hess, F., Englert, U. 2003. First Cr(III)-SNS complexes and their use as highly efficient catalysts for the trimerization of ethylene to 1 hexene. Journal of the American Chemical Society, 125, 5272-5273.

[21] McGuinness, D. S., Wasserscheid, P., Morgan, D. H., Dixon, J. T. 2005. Ethylene trimerization with mixed-donor ligand $(\mathrm{N}, \mathrm{P}, \mathrm{S})$ chromium complexes: effect of ligand structure on activity and selectivity. Organometallics, 24, 552-556.

[22] Downing, S. P., Hanton, M. J., Slawin, A. M. Z., Tooze, R. P. 2009. Bis(alkylthioethyl)amine complexes of molybdenum. Organometallics, 28, 2417-2422.

[23] Shaffer, D. W., Szigethy, G., Ziller, J. W., Heyduk, A. F. 2013. Synthesis and characterization of a redox-active Bis(thiophenolato)amide ligand, $[\mathrm{SNS}]^{3-}$, and the homoleptic tungsten complexes, $\mathrm{W}[\mathrm{SNS}]_{2}$ and $\mathrm{W}[\mathrm{ONO}]_{2}$. Inorganic Chemistry, 52 , 2110-2118.

[24] Sogukomerogulları, H. G., Aytar, E., Ulusoy, M., Demir, S., Dege, N., Richeson, S. D., Sönmez, M. 2017. Synthesis of complexes $\mathrm{Fe}, \mathrm{Co}$ and $\mathrm{Cu}$ supported by "SNS" pincer ligandsn and their ability to catalytically form cyclic carbonates. Inorganica Chimica Acta, 471, 290-296.

[25] Maravalli, P. B., Dhumwad, S. D., Goudar, T. R. 1999. Synthetic, spectral, thermal and biological studies of lanthanide(III) complexes with a Schiff base derived from 3-N-methylpiperidino4-amino-5mercapto-1,2,4-triazole. Synthesis and Reactivity in Inorganic and Metal-Organic Chemistry, 29, 525-540.

[26] Wong, K. N., Colson, S. D. 1984. The FT-IR spectra of pyridine and pyridine- $\mathrm{d}_{5}{ }^{1}$. Journal of Molecular Spectroscopy, 104, 129-151.

[27] Sogukomerogullari, H. G., Şen, F., Dinçer, M., Özdemir, N., Sönmez, M. 2017. Tridentate SNS pincer type ligand: synthesis, structural and spectroscopic analysis of a novel pyridine and m-xylene compound with thioether-bridge. Journal of Molecular Structure, 1136, 271-280.

[28] Miecznikowski, J. R., Lynn, M. A., Jasinski, J. P., Reinheimer, E., Bak, D. W., Pati, M., Butrick, E. E., Drozdoski, A. E. R., Archer, K. A., Villa, C. E., Lemons, E. G., Powers, E., Siu, M., Gomes, C. D., Morio, K. N. 2014. Synthesis, characterization, and computational study of three-coordinate SNS-copper(I) complexes based on bis-thione precursors. Journal of Coordination Chemistry, 67, 29-43.

[29] Zhang, J., Pan, M., Jiang, J., She, Z., Fan, Z., Su, C. 2011. Syntheses, crystal structures and antimicrobial activities of thioether ligands containing quinoline and pyridine terminal groups and their transition metal complexes. Inorganica Chimica Acta, 374, 269-277.

[30] Sogukomerogulları, H. G., Yalçın, Ş. P., Ceylan, Ü., Aytar, E., Aygün, M., Richeson, D. S., Sönmez, M. 2019. Synthesis of $\mathrm{Fe}$ and $\mathrm{Cu}$ metal complexes derived from 'SNS' Pincer type ligands and their efficient catalyst precursors for the chemicaln fixation of $\mathrm{CO}_{2}$. Journal of Chemical Sciences, $131,32-44$.

[31] Sönmez, M., Çelebi, M., Levent, A., Berber, İ., Şentürk, Z. 2010. A new pyrimidine-derived ligand, $\mathrm{N}$-pyrimidine oxalamic acid, and its $\mathrm{Cu}$ (II), Co (II), Mn (II), Ni (II), Zn (II), Cd (II), and Pd (II) complexes: synthesis, characterization, electrochemical properties, and biological activity. Journal of Coordination Chemistry, 63, 848-860.

[32] Bai, S. Q., Quek, G. Y. H., Koh, L. L., Hor, T. S. A. 2010. Crystallographic analysis of different water-halide cluster blends in cationic [(SNS)Pd(II)] pincer complexes. CrystEngComm, $12,226-233$.

[33] Miecznikowski, J. R., Lynn, M. A., Jasinski, J. P., Lo, W., Bak, D. W., Pati, M., Butrick, E. E., Drozdoski, A. E. R., Archer, K. A., Villa, C. E., Lemons, E. G., Powers, E., Siu, M., Gomes, C. D., Bernier, N. A., 
Morio, K. N. 2014. Synthesis and characterization of five-coordinate copper(II) complexes based on tridentate SNS pincer ligand precursors. Polyhedron, 80, 157-165.

[34] Karam, A. R., Catari, E. L., Lopez-Linares, F., Agrifoglio, G., Albano, C.L., DiazBarrios, A., Lehmann, T. E., Pekerar, S. V., Albornoz, L. A.,
Atencio, R., Gonzalez, T., Ortega, H. B., Joskowics, P. 2005. Synthesis, characterization and olefin polymerization studies of iron(II) and cobalt(II) catalysts bearing 2,6-bis(pyrazol-1yl)pyridines and 2,6-bis(pyrazol-1-ylmethyl)pyridines ligands. Applied Catalysis A: General, 280, 165173. 